Europhys. Lett., 74 (6), pp. 999-1005 (2006)

DOI: $10.1209 / \mathrm{epl} / \mathrm{i} 2006-10059-\mathrm{y}$

\title{
Role of mean free path in spatial phase correlation and nodal screening
}

\author{
B. A. van Tiggelen, D. Anache and A. Ghysels(*) \\ Laboratoire de Physique et Modélisation des Milieux Condensés/CNRS \\ Maison des Magistères/UJF, BP 166, 38042 Grenoble, France
}

received 31 January 2006; accepted in final form 25 April 2006

published online 17 May 2006

PACS. 42.25.Dd - Wave propagation in random media.

PACS. 42.30.Rx - Phase retrieval.

PACS. 91.30.Cd - Seismology: Body wave propagation.

\begin{abstract}
We study the spatial correlation function of the phase and its derivative, and related, fluctuations of topological charge, in two- and three-dimensional random media described by Gaussian statistics. We investigate their dependence on the scattering mean free path.
\end{abstract}

Introduction. - In disordered media the scattering mean free path (notation $\ell$ ) denotes the characteristic distance that waves need to travel to achieve a random phase [1]. The length $\ell$ sets the scale in all aspects of multiple scattering. "Mesoscopic" samples have sizes larger than $\ell$ and smaller than the decoherence length, beyond which the phase is irreversibly destroyed, e.g. by coupling to a noisy environment. A third fundamental length, the transport mean free path (notation $\ell^{*}$ ), determines the randomization of the direction of propagation. It is closely related to $\ell$ but the physics is subtly different: whereas $\ell$ is determined by superposition, $\ell^{*}$ is also sensitive to interference. In a more condensed-matter language, $\ell$ is a property of the averaged one-particle Green's function, whereas $\ell^{*}$ is a property of the averaged two-particle Green's function.

Despite its conceptual importance, $\ell$ is rather unaccessible. Theoreticians have found it hard to calculate for dense media because infinitely many "Feynman diagrams" contribute [1], and choices have usually been guided by effective medium arguments [2]. In transport experiments it is mostly $\ell^{*}$ that appears in observables [3]. In electronic systems, $\ell$ is known to govern the magnetic "De Haas" quantum oscillations [4]. Contrary to Sharvin-Sharvin $h / 2 e$ oscillations, Aharonov-Bohm $h / e$ oscillations damp out in loops larger than the scattering length $\ell[5]$. To get $\ell$ of classical waves, we must measure the "coherent" field $\langle\Psi\rangle$, either for different sample thicknesses or for different times. This requires a coherent source, phasesensitive detection of an exponentially small signal, and a good ensemble average. Today, these requirements can be met for ultrasound [6] and electromagnetic waves [7]. A final complication is that coherent beam experiments always measure the combination of phase

$\left({ }^{*}\right)$ Present address: Center for Molecular Modelling, Universiteit Gent - Proeftuinstraat 86, 9000 Gent, Belgium. 
randomization (length $\ell$ ) and absorption (length $\ell_{a}$ ), quantified by the extinction length $\ell_{e}$, with $1 / \ell_{e}=1 / \ell+1 / \ell_{a}$. Here, we investigate the possibility to retrieve the scattering mean free path of a random medium from the spatial phase statistics of the scattered waves, rather than from the coherent field. The phase of a diffuse field can be measured using a "passive" source, whose coherence, position and magnitude are of no relevance, provided it delivers diffuse energy well in excess of the background noise.

First, we introduce the field correlation function in random media and discuss the role of small absorption. Secondly, we discuss the basic results related to phase statistics and give a rapid theoretical support. We finally discuss the relation to topological charge, whose variance has been studied extensively in the literature.

Spatial field correlation. - In a multiply scattering medium far from the localization threshold, it is widely accepted that the complex field obeys circular Gaussian statistics [8]. Since the average field $\langle\Psi(\boldsymbol{r}, t)\rangle$ vanishes if the source is far away, the only parameter governing the wave statistics is the two-point correlation function $\left\langle\Psi\left(\boldsymbol{r}-\frac{1}{2} \boldsymbol{x}, t-\frac{1}{2} \tau\right) \Psi\left(\boldsymbol{r}+\frac{1}{2} \boldsymbol{x}, t+\frac{1}{2} \tau\right)\right\rangle[9]$. We consider a heterogeneous medium in either $2 \mathrm{D}$ or $3 \mathrm{D}$, in which all waves suffer from the same small absorption rate $1 / \tau_{a}$ in energy (absorption length $\ell_{a}=c \tau_{a}$ ). We assume the medium to be large enough to ignore the boundaries.

We consider a wave field $\psi_{B}(\mathrm{r}, t)$ released by a source with spectral density $S(\omega)$. The subscript $B$ refers to a band-filtering in some frequency band $B$ in which diffusion constant $D$ and absorption time $\tau_{a}$ are constant ( $B t>1$ is imposed to resolve the dynamics). Its (ensembleaveraged) spatial correlation at lapse time $t$ in the coda takes the following diffuse form [10]:

$$
\begin{aligned}
\left\langle\Psi_{B}\left(\boldsymbol{r}-\frac{1}{2} \boldsymbol{x}, t-\tau / 2\right) \Psi_{B}\left(\boldsymbol{r}+\frac{1}{2} \boldsymbol{x}, t+\tau / 2\right)^{*}\right\rangle \\
\sim \frac{\exp \left[-\frac{r^{2}}{4 D t}-\frac{t}{\tau_{a}}\right]}{(D t)^{d / 2}} \int_{B} \frac{\mathrm{d} \omega}{2 \pi} S(\omega) \exp [-i \omega \tau] \operatorname{Im} G\left(\boldsymbol{x}, \omega, \ell_{e}^{-1} \rightarrow \ell^{-1}\right) .
\end{aligned}
$$

This formula shows that, once absorption has been acknowledged by the factor $\exp \left[-t / \tau_{a}\right]$, the field correlation function relates to (the imaginary part of) the absorption-free Green function. This is best understood in the frequency domain, where eq. (1) gives rise to the following product of exponentials:

$$
\exp \left[i\left(\omega+i / 2 \tau_{a}\right)(t-\tau / 2)\right] \times \exp \left[i\left(\omega+i / 2 \tau_{a}\right)(t+\tau / 2)\right]^{*}=\exp [-i \omega \tau] \exp \left[-t / \tau_{a}\right],
$$

from which we infer that the absorption only appears as a function of the lapse time $t$, and not of the local correlation time $\tau$. A first observation is thus that in the diffuse time-tail, we can consider the (normalized) spatial correlation of the field to be free of absorption.

In this work we shall ignore the effects of source spectrum and finite bandwidth, which can both straightforwardly be included into Gaussian statistics [11]. If we denote by $C(x)$ the spatial field correlation function at frequency $\omega[12]$, in the vicinity of some point $\boldsymbol{r}$, deep in the coda at time $t$, and normalized to $C(0)=1$, we find it to be solely dependent on the local Green's function, a central issue in recent attempts to image passively [13]. Contrary to chaotic media, the $C(x)$ of random media always decays exponentially due to dephasing from scattering. In $3 \mathrm{D}$ is $C(x)=\operatorname{sinc}(k x) \exp [-x / 2 \ell]$, whereas in $2 \mathrm{D} C(x)=$ $J_{0}(k x) \exp [-x / 2 \ell][14]$, with $k=2 \pi / \lambda$ the wave number. The damped oscillations on the scale of the wavelength $\lambda$ originate from a superposition of plane waves incident with arbitrary directions but with equal amplitude. They prevent us from seeing the genuine dephasing that occurs on the much longer scale of the scattering mean free path. 
Phase correlation. - Rather than the field correlation, we propose here to consider the spatial phase correlation $\Xi_{B}(\boldsymbol{x}, t) \equiv\left\langle\Phi\left(\boldsymbol{r}-\frac{1}{2} \boldsymbol{x}, t\right) \Phi\left(\boldsymbol{r}+\frac{1}{2} \boldsymbol{x}, t\right)\right\rangle$. The phase $\Phi(\boldsymbol{r}, t)$ is defined as the complex phase of the wave function $\Psi_{B}=A \exp [i \Phi]$. At any point $\boldsymbol{x}$ the phase is a flat random number between $-\pi$ and $\pi$. Upon moving to another point $\boldsymbol{x}^{\prime}$ it will exhibit discontinuous jumps of $2 \pi$ between $\pm \pi$. An occasional jump of a $\pi$ could occur when a singularity with either positive or negative topological charge [15] is crossed, but such event has zero probability on a $1 \mathrm{D}$ line. Let us now "unwrap" the phase, i.e. correct by hand for the $2 \pi$ discontinuities as was done in the frequency domain [16] and in the time domain [17], to get the unwrapped phase or cumulative phase $\Phi_{U}$ that varies continuously with $\boldsymbol{x}$, and which in principle can take all values $[-\infty, \infty]$. This continuation is not topologically invariant, but depends on the path chosen to go from $\boldsymbol{r}$ to $\boldsymbol{r}+\boldsymbol{x}$. Gaussian statistics demonstrate that both the distribution $P(\mathrm{~d} \Phi / \mathrm{d} x)$, and the spatial correlation function $C_{\Phi}(\Delta x)$ of the phase derivative $\mathrm{d} \Phi / \mathrm{d} x$ are smooth, confirming that phase jumps do not affect phase statistics within an infinitesimal increment $\mathrm{d} x$. So the integral of $\mathrm{d} \Phi / \mathrm{d} x$ along the path should — statistically speaking - be equal to the continuous cumulative phase. If we agree to walk straight along the $x$-axis, this results in

$$
\Xi_{U}(x)=\int_{0}^{-x / 2} \mathrm{~d} x^{\prime} \int_{0}^{x / 2} \mathrm{~d} x^{\prime \prime}\left\langle\frac{\mathrm{d} \Phi}{\mathrm{d} x}\left(x^{\prime}\right) \frac{\mathrm{d} \Phi}{\mathrm{d} x}\left(x^{\prime \prime}\right)\right\rangle=-\int_{0}^{x / 2} \mathrm{~d} x^{\prime} x^{\prime}\left[C_{\Phi}\left(x^{\prime}\right)+C_{\Phi}\left(x-x^{\prime}\right)\right] .
$$

The second equality uses that the phase derivative correlation function $C_{\Phi}\left(x^{\prime}, x^{\prime \prime}\right)$ depends only on $\left|x^{\prime}-x^{\prime \prime}\right|$.

The variance of the unwrapped phase has already been discussed in the literature in view of its close connection with fluctuations in the positions of zeros of the wave function [11,18-20]. It follows from eq. (2) that

$$
\left\langle\Phi_{U}^{2}(x)\right\rangle=2 \int_{0}^{x} \mathrm{~d} x^{\prime}\left(x-x^{\prime}\right) C_{\Phi}\left(x^{\prime}\right) .
$$

The phase derivative correlation function $C_{\Phi}(x)$ can be calculated from the joint probability function of 4 complex fields at 4 different positions. This is a circular Gaussian distribution [8], containing 4 amplitudes and 4 phases. The amplitudes can be integrated out, and the four phases can be manipulated into two phase derivatives at different spots. The result for $C_{\Phi}(x)$ is an integral that involves the field correlation $C(x)$, and its derivatives $C^{\prime}(x)$ and $C^{\prime \prime}(x)$ in just the same way as was seen for frequency correlations [21],

$$
\begin{aligned}
C_{\Phi}(x) & =\frac{2}{\pi} \int_{0}^{\pi} \mathrm{d} \phi\left[-\left(C^{\prime \prime}+\frac{C\left(C^{\prime}\right)^{2}}{1-C^{2}}\right) \cos \phi H(C \cos \phi)+\frac{\left(C^{\prime}\right)^{2}}{1-C^{2}} \sin ^{2} \phi H^{\prime}(C \cos \phi)\right] \\
& =\frac{1}{2}(\log C)^{\prime \prime} \log \left(1-C^{2}\right)
\end{aligned}
$$

with $H(y) \equiv \arctan (\sqrt{1+y} / \sqrt{1-y}) / \sqrt{1-y^{2}}$. In the second equality we obtain an analytical expression for the integral. Already for $x>\lambda, C_{\Phi}(x)$ approaches its asymptotic limit,

$$
C_{\Phi}(x>\lambda) \rightarrow \frac{1}{2}\left[\left(C^{\prime}\right)^{2}-C^{\prime \prime} C\right]=\left\{\begin{array}{cc}
(k / \pi x) \exp [-x / \ell] & (2 \mathrm{D}), \\
\left(1 / 2 x^{2}\right) \exp [-x / \ell] & (3 \mathrm{D}) .
\end{array}\right.
$$

We see that, contrary to the field correlation $C, C_{\Phi}$ exhibits no oscillations on the scale of the wavelength. Equation (5) shows that the mean free path $\ell$ assures convergence of eq. (2) in both dimensions, making $\ell$ the characteristic length scale in spatial phase correlation. In fig. 1 we plot $\Xi_{U}(x)$ as a function of $x / \ell$ for $2 \mathrm{D}$. For all values of $k \ell$ it follows a universal 


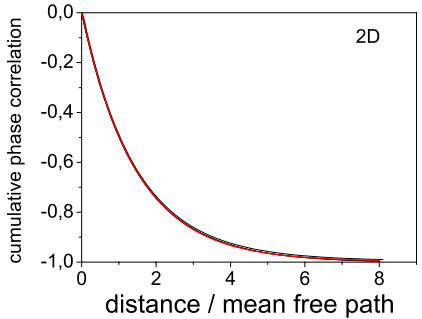

Fig. 1

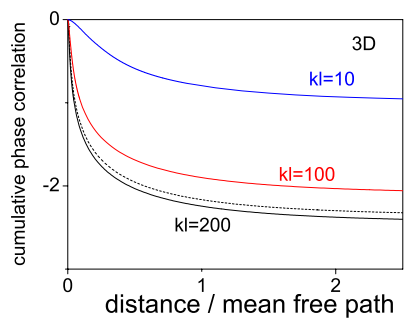

Fig. 2

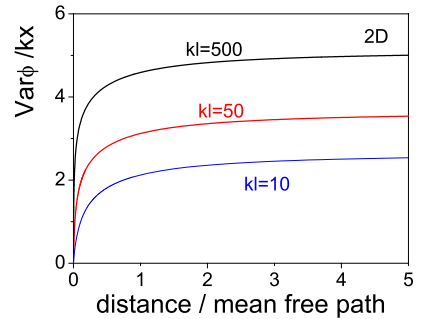

Fig. 3

Fig. 1 - Spatial cumulative (unwrapped) phase correlation $\Xi_{U}(x) v s$. distance $x$ for a random medium in two dimensions. The distance has been measured with respect to the scattering mean free path $\ell$, and the correlation function has been normalized to $k \ell / \pi$ which corresponds to (minus) the asymptotic value for large $k R$. The curves for $k \ell=10,100,200$ overlap and follow an exponential decay (in red on-line).

Fig. 2 - Spatial cumulative phase correlation $\Xi_{U}(x)$ for a random 3D medium, for different values of $k \ell$. The asymptotic value varies logarithmically with $k \ell$. The dashed line is obtained by integrating just the asymptotic formula (5).

Fig. 3 - Cumulative phase variance $\left\langle\Phi_{U}^{2}(x)\right\rangle$ in $2 \mathrm{D}$ random media, divided by the optical path $k x$, against distance $x$, measured in units of the mean free path. Two length scales exist. An initial linear rise is visible on the scale of the wavelength. Subsequently, the phase variance reaches the diffuse plateau after a few mean free paths. The plateau value depends logarithmically on the value $k \ell$.

exponential decay towards the asymptotic value $-k \ell / \pi$. In $3 \mathrm{D}$ the asymptotic value varies only logarithmically with $k \ell$ in 3D (fig. 2) and two characteristic length scales appear, $\lambda$ and $\ell$. A measurement of $\Xi_{U}(x)$ would thus give us direct access to $k \ell$ and $\ell$ in both cases.

The same study has been performed for the phase variance. We infer from eq. (3) that the parameter $\left\langle\Phi_{U}^{2}(x)\right\rangle / x$ is less sensitive to large values for $x$ than the correlation $\Xi_{U}(x)$. In $2 \mathrm{D}$ random media (fig. 3), its plateau value $D_{\Phi}$ (the "phase diffusion constant") depends logarithmically on $k \ell$, although the diffuse plateau is still reached after typically one mean free path. In 3D (dashed line in fig. 4) we see that after an approximate linear rise, $\left\langle\Phi_{U}^{2}(x)\right\rangle / x$ converges already after a few wavelengths to the asymptote $D_{\Phi}=1.922 \times k$, independent of $k \ell$. We conclude that the cumulative phase correlation is sensitive to the mean free path in both $2 \mathrm{D}$ and $3 \mathrm{D}$, unlike the phase variance which depends on $\ell$ only in $2 \mathrm{D}$.

Statistics of topological charge. - It was realized recently by Wilkinson [19] that the spatial phase correlation function is a, key element in an old discussion on the statistics of topological charge of Gaussian field inside a closed contour. This discussion goes back to Halperin [22] and Nye and Berry [9], with significant contributions later by Berry and Dennis [11], and Freund and Wilkinson [18]. The zeros of a complex function in $d$ dimensions are located on $d-2$ "nodal" hypersurfaces, nodal lines in 3D and nodal points in 2D. The topological charge $Q$ enclosed by a 2D surface is defined as the number of nodal points weighed by their topological sign. This number is determined by the order of the vortex surrounding the nodal point [15]. For complex Gaussian random fields topological charges different from \pm 1 are highly improbable [9]. The following relation holds:

$$
\oint_{\Gamma} \mathrm{d} \boldsymbol{r} \cdot \nabla \Phi(\boldsymbol{r})=2 \pi Q
$$




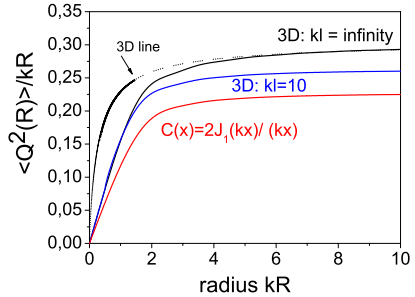

Fig. 4

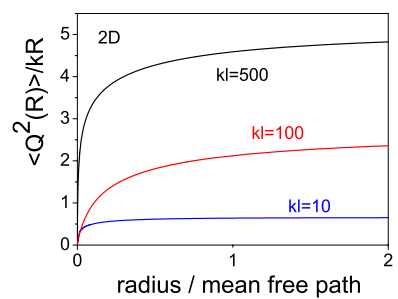

Fig. 5

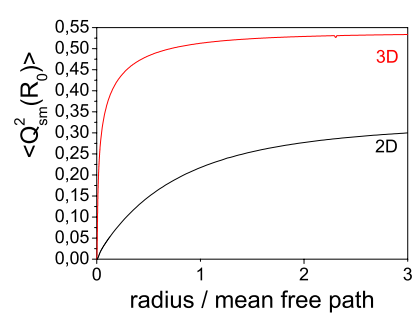

Fig. 6

Fig. 4 - Fluctuations of the topological charge accumulated inside a circle of radius $R$ of a Gaussian random field. On the vertical axis is plotted the variance of $\left\langle Q^{2}\right\rangle$ divided by $2 \pi R / \lambda$, i.e. the circumference in units of the wavelength. Upper two solid lines: $3 \mathrm{D}$ random medium with $k \ell=\infty, 10$. The dashed curve is the cumulative phase variance — divided by $2 \pi$, see eq. (6) - obtained by walking along a straight line with length $2 \pi R$. The bottom solid curve is calculated for a field correlation function $C(x)=2 J_{1}(k x) / k x[15]$. In all cases the characteristic length scale is the wavelength, and not the mean free path.

Fig. 5 - Fluctuations of the topological charge in $2 \mathrm{D}$, accumulated inside a circle of radius $R$. On the vertical axis is plotted the variance of $\left\langle Q^{2}\right\rangle$ divided by the circumference $2 \pi R / \lambda$ in units of the wavelength. On the horizontal axis the radius of the circle is normalized by the scattering mean free path. The characteristic length scale in $2 \mathrm{D}$ is the mean free path. The variance of the topological charge depends logarithmically on the value $k \ell$.

Fig. 6 - Variance of the smoothed topological charge in 3D (normalized to $k \ell$ ) and in 2D (normalized to $\log k \ell)$. The smoothing is done on $Q(R)$ with the Gaussian $\exp \left[-R^{2} / R_{0}^{2}\right]$, as a function of $R_{0}$ in units of the scattering mean free path. For $R_{0}>\ell$ it converges to a constant number dependent on dimension.

with $\Gamma$ the line contour enclosing the surface. This relation is reminiscent of the quantized rotation of superfluids, and is deeply related to the Rouché theorem in complex analysis. Its validity can be understood by applying Stokes' theorem to the function $\nabla \log \psi(\boldsymbol{r})$, whose imaginary part has been analytically continued along the contour.

Equation (6) facilitates a study of the statistics of the topological charge $Q$. In the diffuse regime the phase gradient in any direction vanishes on average, so $\langle Q\rangle=0$. Following Wilkinson [19], we will relate the variance of $Q$ to the correlation of the phase derivative. More precisely, if $\theta \times R$ measures the length along a circular contour with radius $R$ we find

$$
\left\langle Q^{2}(R)\right\rangle=\frac{1}{(2 \pi)^{2}} \int_{0}^{2 \pi} \mathrm{d} \theta \int_{0}^{2 \pi} \mathrm{d} \theta^{\prime} C_{\Phi}(\Delta \theta)=\frac{1}{2 \pi} \int_{0}^{2 \pi} \mathrm{d} \Delta \theta C_{\Phi}(\Delta \theta) .
$$

The phase derivative correlation $C_{\Phi}(\Delta \theta) \equiv\left\langle\partial_{\theta} \Phi(\theta) \partial_{\theta} \Phi\left(\theta^{\prime}\right)\right\rangle$ is, mutadis mutandis, given by eq. (4) (with $\left(^{\prime}\right)=\partial_{\Delta \theta}$ and $C=C[2 k R \sin (\Delta \theta / 2)]$ ). One basic feature of $\left\langle Q^{2}(R)\right\rangle$ is already known. If we assumed all nodal points $n$ to have random charges $Q_{n}= \pm 1$, independent of each other, we would find $\left\langle\left(\sum Q_{n}\right)^{2}\right\rangle \sim R^{2}$, i.e. proportional to the surface. Actually, they are not independent but tend to be perfectly screened [11,22], which affects the quadratic law. We shall define the screening length $\xi$ as the typical length beyond which the asymptotic form of the charge variance is reached. Wilkinson and Freund report a linear, "diffuse" asymptotic form [15],

$$
\lim _{R \rightarrow \infty} \frac{\left\langle Q^{2}(R)\right\rangle}{R}=\frac{1}{\pi} \int_{0}^{\infty} \mathrm{d} x \frac{C^{\prime}(x)^{2}}{1-C(x)^{2}}
$$


We have verified that eq. (7) is consistent with this diffusion law, with the same phase diffusion constant obtained earlier for the phase accumulated along a straight line (figs. 3 and 4). Berry and Dennis [11] calculate $\left\langle Q^{2}(R)\right\rangle$ from the topological charge correlation function, which is, due to screening, sensitive to "edge effects". They smooth the charge distribution with the Gaussian $\exp \left[-R^{2} / R_{0}^{2}\right]$, and consider the variance of total smoothed charge (total integral over $R$ ) associated with the smoothed surface $\pi R_{0}^{2}$. For small $R_{0}$, the quadratic law is still valid but for large $R_{0}$ a behavior different from eq. (8) is found,

$$
\lim _{R_{0} \rightarrow \infty}\left\langle Q_{\mathrm{sm}}^{2}\left(R_{0}\right)\right\rangle=\int_{0}^{\infty} \mathrm{d} x x \frac{C^{\prime}(x)^{2}}{1-C(x)^{2}}
$$

provided the integral converges. In figs. 4 and 5 we have calculated $\left\langle Q^{2}\right\rangle$ for a disk in $2 \mathrm{D}$ and 3D random media. For small $k R$ we see that $\left\langle Q^{2}\right\rangle \sim k^{2} R^{2}$ for both dimensions. In 3D $\left\langle Q^{2}\right\rangle$ at large $R$ depends very weakly on the mean free path $\ell$ since the integral in eq. (8) converges even for $\ell=\infty$. For the field correlation $C(x)=2 J_{1}(k x) / k x$ [15] the curve is very similar though with a somewhat smaller phase diffusion constant. In both cases this yields a screening length proportional to the wavelength. For 2D random media we can see that $\left\langle Q^{2}\right\rangle$ depends logarithmically on $k \ell$. The topological screening length now depends on the mean free path. The variance of smoothed charge $\left\langle Q_{\mathrm{sm}}^{2}\right\rangle$ however (fig. 6), converges to $k \ell / \pi$ in $2 \mathrm{D}$ and to $0.54 \log k \ell$ in $3 \mathrm{D}$, with a screening length typically equal to the $\ell$. Note that in $2 \mathrm{D}$ chaotic media, for which $C(x)=J_{0}(k x)$, the integrals (8) and (9) both diverge and the topological charge variance increases as $\left\langle Q^{2}\right\rangle \propto k R \log (k R)$, i.e. it is slightly superdiffuse, whereas its smoothed version $\left\langle Q_{\mathrm{sm}}^{2}\right\rangle$ no longer reaches a constant but becomes diffuse: $\left\langle Q_{\mathrm{sm}}^{2}\right\rangle \rightarrow k R_{0} /(2 \sqrt{\pi})$.

Conclusions. - We have shown that in the late coda of waves propagating in $2 \mathrm{D}$ and $3 \mathrm{D}$ random media, the scattering mean free path governs the spatial fluctuations of both the phase derivative $\mathrm{d} \Phi / \mathrm{d} x$, cumulative phase and the topological charge, measured inside a surface of size $A$. Screening makes topological charge fluctuations grow slower than $A$. The screening length is defined as the typical length beyond which they reach their final behavior. In $2 \mathrm{D}$ it is proportional to the mean free path, in $3 \mathrm{D}$ to the wavelength. If the charge is smoothed with a Gaussian, the screening length is typically equal to the mean free path, in both dimensions. This highlights the subtle role of smoothing screened charge.

The spatial correlation of $\mathrm{d} \Phi / \mathrm{d} x$, whose measurement requires only four phase-sensitive detectors, decays exponentially with the scattering mean free path $\ell$ as the sole length scale. In seismic measurements, often dominated by 2D Rayleigh waves, this may be a novel opportunity to measure the scattering mean free path of surface waves. Such measurement would not be sensitive to absorption, neither to the precise source location, quite opposed to a measurement of the coherent beam.

$$
* * *
$$

The authors would like to thank L. Margerin, A. Genack, R. Weaver, J. Page and M. Dennis for useful discussions.

\section{REFERENCES}

[1] Frisch U., Probabilistic Methods in Applied Mathematics, Vols. I, II, edited by BharuchaReID A. T. (Academic, New York) 1968.

[2] Busch K. and Soukoulis C. M., Phys. Rev. Lett., 75 (1995) 3442; Page J. M., Sheng P., Schriemer H. P., Jones I., Jing W. and Weitz D. A., Science, 271 (1996) 634; Kirchner A., Busch K. and Soukoulis C. M., Phys. Rev. B, 57 (1998) 277. 
[3] Aknermans E. and Montambaux G., Physique Mésoscopique des Electrons et des Photons (EDP Sciences) 2004.

[4] See, e.g., ref. [3], sect. 14.2.2.

[5] Washburn S. and Webb R. A., Adv. Phys., 35 (1986) 375; Vloerbergs H. et al., Phys. B, 175 (1991) 217.

[6] Derode A., Tourin A. and Fink M., Phys. Rev. E, 64 (2001) 036605; Scales J. A. and Malcolm A. E., Phys. Rev. E, 67 (2003) 0466181-7.

[7] See, e.g., Kempe M., Genack A. Z., Rudolf W. and Dorn P., J. Opt. Soc. Am. A, 14 (1997) 216.

[8] Goodman J. W., Statistical Optics (Wiley) 1985.

[9] Nye J. F. and Berry M. V., Proc. R. Soc. London, Ser. A, 336 (1974) 165.

[10] Barabanenkov Yu. N. and Ozrin V. D., Phys. Lett. A, 206 (1995) 116.

[11] Berry M. V. and Dennis M. R., Proc. R. Soc. London, Ser. A, 456 (2000) 2059; Dennis M. R., J. Phys. A, 36 (2003) 6611.

[12] Sebbah P., Pnini R. and Genack A. Z., Phys. Rev. E, 62 (2000) 7348; Sebbah P., Hu B., Genack A. Z., Pnini R. and Shapiro B., Phys. Rev. Lett., 88 (2002) 123901.

[13] Weaver R. and Lobkis O., Ultrasonics, 40 (2003) 435; Campillo M. and Paul A., Science, 299 (2003) 547; Derode A. et al., J. Acoust. Soc. Am., 113 (2003) 2973; Snieder R. K., Phys. Rev. E, 69 (2004) 046610; Malcolm A. E., Scales J. A. and Van Tiggelen B. A., Phys. Rev. E, 70 (2004) 015601.

[14] One can show that $C^{\prime}(0)=0$ and $C^{\prime \prime}(0)=-\frac{1}{d} k^{2} c_{0} / v_{E}$ with $v_{E}$ the transport velocity. The exponential decay proposed for $C(x)$ is not valid near $x=0$, and only correct for large $x$. In this work we exclude the exponential factor for $x<\lambda$, and also ignore phase accumulation inside the scatterers, which is the reason why $v_{E}$ should appear.

[15] Freund I. and Shvartsman N., Phys. Rev. E, 51 (1995) 3770; Freund I., Phys. Rev. E, 52 (1995) 2348.

[16] Sebbah P., Legrand O., Van Tiggelen B. A. and Genack A. Z., Phys. Rev. E, 56 (1997) 3619.

[17] Page J. H., Cowan M. L., Weitz D. A. and Van Tiggelen B. A., Wave Scattering in Complex Media: From Theory to Applications, edited by Skipetrov S. E. and Van Tiggelen B. A. (Kluwer, Dordrecht) 2003.

[18] Freund I. and Wilkinson M., J. Opt. Soc. Am. A, 18 (1998) 2892.

[19] Wilkinson M., J. Phys. A, 37 (2004) 6763.

[20] Foltin G., J. Phys. A, 36 (2003) 4561.

[21] Van Tiggelen B. A., Sebbah P., Stoytchev M. and Genack A. Z., Phys. Rev. E, 59 (1999) 7166.

[22] Halperin B. I., Statistical Mechanics of Topological Defects, Les Houches Lectures XXVPhysics of Defects, edited by Balian R., Kléman M. and Poirier J. P., Vol. 35 (NorthHolland, Amsterdam) 1981. 